\title{
Suppression of lung cancer cell invasion by LKB1 is due to the downregulation of tissue factor and vascular endothelial growth factor, partly dependent on SP1
}

\author{
XUAN LIANG $^{1 *}$, ZHAO-LUN LI $^{2 *}$, LI-LI JIANG ${ }^{1}$, QIAN-QIAN GUO ${ }^{1}$, MENG-JIE LIU ${ }^{1}$ and KE-JUN NAN ${ }^{1}$ \\ ${ }^{1}$ Department of Oncology, First Affiliated Hospital; ${ }^{2}$ Department of Urology, Second Affiliated Hospital, \\ Xi'an Jiaotong University Medical College, Xi'an, Shaanxi 710061, P.R. China
}

Received December 22, 2013; Accepted February 19, 2014

DOI: $10.3892 /$ ijo.2014.2351

\begin{abstract}
LKB1 encodes a serine/threonine kinase generally inactivated in many human cancers, which mediates cancer cell proliferation, migration and differentiation. Recent studies indicated that LKB1 exhibits potent anti-metastatic activity. However, the underlying molecular mechanisms of this activity remain unclear. In this study, we re-introduced LKB1 into A549 lung cancer cells that lack the LKB1 gene to investigate how LKB1 affects tumor invasiveness and metastasis. We demonstrated that overexpression of the LKB1 protein in lung cancer cells resulted in significant inhibition of invasion. Furthermore, transfected lung cancer cells with LKB1 suppressed tissue factor (TF) and vascular endothelial growth factor (VEGF) expression at both the mRNA and protein levels. Here, we provided evidence showing that downregulation of TF and VEGF by LKB1 is correlated well with the inhibition of cell invasion. Overexpression of the LKB1 protein in human lung cancer is significantly associated with a decrease in activity and expression of the transcription factor SP1. Constitutive activation of the transcription factor Sp1 plays a critical role in TF and VEGF overexpression. We conclude that suppression of lung cancer cell invasion by LKB1 through downregulation of TF and VEGF may partly depend on its inhibitory effect on the transcription factor $\mathrm{Sp} 1$. Collectively, our data provide a novel molecular mechanism for the antitumor activity of LKB1 and may help further improve its effectiveness in controlling lung cancer growth and invasion.
\end{abstract}

Correspondence to: Professor Ke-Jun Nan, Department of Oncology, First Affiliated Hospital, Xi'an Jiaotong University Medical College, Xi'an, Shaanxi 710061, P.R. China

E-mail: nankj1958@163.com

*Contributed equally

Key words: LKB1/STK11, A549 cells, invasion, tissue factor, vascular endothelial growth factor

\section{Introduction}

Lung cancer is one of the most common malignancies and the development of clinical metastasis is the main cause of morbidity and mortality (1). Tumor invasion plays a crucial role in metastasis and involves a number of important steps, including adhesion of tumor cells to the basement membrane (BM); enzymatic digestion of the BM by proteolytic enzymes followed by migration through the extracellular matrix (ECM) with the subsequent growth and proliferation of cells at a new site $(2,3)$. Any agent, which can inhibit the invasive process, may therefore become a powerful tool in the prevention of metastasis.

LKB1 (also known as STK11) is a recently identified tumor suppressor gene whose mutation can lead to Peutz-Jeghers syndrome, which is characterized by gastrointestinal polyps and cancers of different organ systems. The functional loss of LKB1 or LKB1-inactivating mutations are known to be correlated with one-third of sporadic lung adenocarcinomas $(4,5)$, indicating that LKB1 gene is related to the tumorigenesis of lung cancer. Recent reports have suggested that LKB1 is critical for cell migration leading to invasion and metastasis of cancer (6). However, the molecular mechanisms of these multiple biological events throughout the process of tumor development and progression are largely unknown.

To further investigate whether and how LKB1 affects the migration and invasion of lung cancer cells, we generated an LKB1 overexpression cell line, A549/LKB1 cells. LKB1 was examined for its potential on lung cancer cell invasion and related molecular mechanisms in A549 cells. Our previous studies showed that LKB1 exerted tumor inhibitory effects on human lung carcinoma cells in vitro (7-9). The mechanisms of these inhibitory effects may include the upregulation or downregulation of the different effector molecules. Here we provide evidence showing that LKB1 suppresses tissue factor (TF) and vascular endothelial growth factor (VEGF) expression, which is correlated well with the inhibition of cell invasion. VEGF is the most powerful endothelial cell specific mitogens associated with tumor neovascularization. It has been reported that LKB1 is involved in the VEGF signaling pathway and that the vascular defects accompanying LKB1 loss are related with VEGF $(10,11)$. The expression of VEGF 
regulated by LKB1 is likely to play a contributory role in tumor cells invasion. TF, an initiator of the extrinsic coagulation cascade, is also expressed in a wide range of cancer cells. It is known that $\mathrm{TF}$ promotes angiogenesis by cooperating with VEGF in several malignant tumors $(12,13)$. Koomagi and Volm demonstrated that TF was related to VEGF expression and microvessel density and that the survival times were longer in patients whose tumors were immunologically TF-negative than in those with TF-positive tumors (14). Given that tumor cells sometimes mimic normal physiological systems for the purpose of invasion and progression, TF may also contribute to its promotion of cancer metastasis and progression. However, how LKB1 gene regulates those different effector molecules becomes an increasingly important question. The answer will not only shed light on the mechanism of cancer invasion and metastasis but also likely lead to clinical benefits.

\section{Materials and methods}

Cell culture and transfection. The LKB1-null human lung cell line A549 cells were plated in culture plates in Dulbecco's minimal essential medium (DMEM, Gibco, Grand Island, NY, USA) containing $10 \%(\mathrm{v} / \mathrm{v})$ fetal bovine serum (FBS, Gibco). The cultures were maintained at $37^{\circ} \mathrm{C}$ in a humidified atmosphere of $5 \% \mathrm{CO}_{2}$. After culture to $70 \%$ confluence on 6 -well plates, cells were transfected with pcDNA/LKB1 or control (vehicle) pcDNA3.1 (the vectors containing Neomicin/G418 resistance gene were kindly provided by Dr Qingzhi Xu) in the presence of Lipofectamine 2000 (Invitrogen Corp., Grand Island, NY, USA) following the manufacturer's protocol. Transfection medium was replaced with growth medium containing $10 \%$ FBS after cells were incubated with transfection reagents for $6 \mathrm{~h}$. Then, day 16 after selection with complete DMEM containing $600 \mu \mathrm{g} / \mathrm{ml} \mathrm{G} 418$ (Gibco), all untransfected cells died and discrete clones were visible in transfected cells. These clones were expanded in the presence of $300 \mu \mathrm{g} /$ ml G418 to be used for the further study. Cells transfected with pcDNA/LKB1 and pcDNA3.1 vectors were termed A549/LKB1 and A549/vec, respectively. Both transfection and G418 selection were conducted under sterile conditions and duplicate plates were tested for each condition. The full-length human Sp1 AN cDNA was made by cloning EcoRI restriction fragments from the plasmid pBS-Sp1-AN (a kind gift from Dr J. Marvel and Dr Y. Leverrier, France) and was inserted into EcoRI sites of pcDNA3.1.

$R T-P C R$. Total RNA was isolated from the cells using TRIzol reagent (Invitrogen) according to the procedure provided by the manufacturer. Reverse transcription was performed from total RNA at $42^{\circ} \mathrm{C}$ for 60 min by M-MLV Reverse Transcriptase (RNase-free, Takara, Shiga, Japan). PCR amplification was performed to synthesize these gene products, and the products were analyzed by electrophoresis on a $1.5 \%$ agarose gel. The ratio of the yield of mRNA to the yield of the relevant internal standard [glyceraldehyde-3-phosphate dehydrogenase (GAPDH)] was calculated and compared. The specific primers and their annealing temperatures are listed in Table I.

Western blot analysis. Total cell lysates were prepared using the cell lysis buffer (Pierce, Rockford, IL, USA) following the manufacturer's instructions. Total protein was estimated using BCA analysis (Pierce). The samples were re-suspended in SDS-PAGE loading buffer and heated at $100^{\circ} \mathrm{C}$ for $5 \mathrm{~min}$. Equal amounts of protein were loaded on gel and then transferred to nitrocellulose membrane (Mini-Protean and Trans-Blot systems, Bio-Rad Laboratories, Hercules, CA, USA). The nitrocellulose membrane was blocked with 5\% non-fat dry milk and separately incubated with corresponding specific primary antibodies, including 1:800 rabbit anti-LKB1 (Cell Signaling Technology, Danvers, MA, USA), 1:1,500 mouse anti-actin, 1:750 mouse anti-TF (AbD Serotec, Oxford, UK), 1:500 goat anti-VEGF (Santa Cruz Biotechnology, Santa Cruz, CA, USA), 1:1000 mouse anti-SP1 (Santa Cruz Biotechnology). After washing and incubating with corresponding secondary antibodies conjugated horseradish peroxidase (1:2,000 dilution of goat anti-rabbit IgG antibody, 1:1,500 dilution of rabbit anti-goat IgG antibody, 1:2,000 dilution of goat anti-mouse IgG antibody, respectively). The proteins were detected by chemiluminescence and exposure to light-sensitive film.

In vitro adhesion assays. Cell adhesiveness to the extracellular matrices was measured by an adhesion assay. The 96-well culture plates were precoated with $50 \mu$ l Matrigel (diluted 1:8 in complete DMEM cell culture medium) and dried overnight at $4^{\circ} \mathrm{C}$. The wells were then rehydrated and blocked for $1 \mathrm{~h}$ at $37^{\circ} \mathrm{C}$ with $1 \%$ bovine serum albumin (BSA) in serum-free DMEM cell culture medium. Cells were seeded at a density of $1 \times 10^{5}$ cells/well on the Matrigel-coated 96-well plates and allowed to adhere for $1 \mathrm{~h}$ in a $37^{\circ} \mathrm{C}$ humid chamber. After incubation, non-adherent cells were carefully removed by washing gently with PBS. After stained for $4 \mathrm{~h}$ at $37^{\circ} \mathrm{C}$ with $10 \mu \mathrm{l}$ MTT $(5 \mathrm{mg} / \mathrm{ml})$, adherent cells were diluted in $150 \mu \mathrm{l}$ of DMSO and measured at $570 \mathrm{~nm}$ using a microtiter plate reader (Wako, Shanghai, China). All experiments were performed in triplicate. The adhesion index was calculated as the percentage ratio between the number of adherent test cells and the number of control cells.

In vitro migration assays. The Boyden chamber procedure was used to evaluate cell migration activity in a 24-microwell chamber (Millipore, Billerica, MA, USA). The upper and lower wells were separated by a polyvinyl-pyrolidone-free polycarbonate filter $(8-\mu \mathrm{m}$ pore size). The lower wells contained DMEM with $10 \%$ FBS. Cell suspension $\left(2.5 \times 10^{4}\right.$ cells in $100 \mu \mathrm{l}$ of serum-free medium) of A549 cells and A549/LKB1 cells (transfected with LKB1), was added to the upper wells, respectively. The chamber was incubated at $37^{\circ} \mathrm{C}$ for $24 \mathrm{~h}$. Non-migrating cells on the upper surface of the filters were removed with a cotton swab. The filters were then removed and fixed in $95 \%$ ethanol for $10 \mathrm{~min}$. Cells were stained with $0.5 \%$ crystal violet in $20 \%$ ethanol and counted using a light microscope in 10 random fields per each well. Migration was assayed by measuring the number of cells moving across the filter. The number of cells that migrated is given as the mean $\pm \mathrm{SD}$. Each experiment was performed in triplicate.

In vitro invasion assays. The Matrigel invasion assay (BD Biosciences, Heidelberg, Germany) was used to assess the invasive potential of A549 lung cancer cells. Briefly, BioCoat Matrigel invasion chambers were rehydrated according to 
Table I. Primers and annealing temperatures.

\begin{tabular}{|c|c|c|c|}
\hline Gene & Specific primers & $\begin{array}{c}\text { Annealing } \\
\text { temperature }\left({ }^{\circ} \mathrm{C}\right)\end{array}$ & $\begin{array}{l}\text { Product } \\
\text { length (bp) }\end{array}$ \\
\hline LKB 1 & $\begin{array}{l}5^{\prime}>\text { CGGCAAGGTGAAGGAA }<3 ' \\
5^{\prime}>\text { ACGCCCAGGTCGGAGAT }<3^{\prime}\end{array}$ & 55 & 411 \\
\hline SP1 & $\begin{array}{l}5^{\prime}>\text { AACCCACAAGCCCAAACAATCACC }<3^{\prime} \\
5^{\prime}>\text { CCCCGAGCCCCTTCCTTCACT }<3^{\prime}\end{array}$ & 60 & 428 \\
\hline $\mathrm{TF}$ & $\begin{array}{l}\text { 5'>TGAAGGATGTGAAGCAGACG }<3 \text { ' } \\
\text { 5'>TGAAGGATGTGAAGCAGACG }<3 \text { ' }\end{array}$ & 60 & 451 \\
\hline VEGF & $\begin{array}{l}\text { 5'>TGGATCCATGAACTTTCTGCTGTC< }<\text { ' } \\
\text { 5'>TCACCGCCTTGGCTTGTCACAT<3' }\end{array}$ & 57 & $\begin{array}{l}542 \\
528\end{array}$ \\
\hline GAPDH & $\begin{array}{l}5^{\prime}>\text { ACCACAGTCCATGCCATCAC }<3^{\prime} \\
5^{\prime}>\text { TCCACCACCCTGTTGCTGTA }<3^{\prime}\end{array}$ & 60 & 452 \\
\hline
\end{tabular}

the manufacturer's instructions. Following rehydration, the medium was carefully removed. DMEM cell culture medium (500 $\mu \mathrm{l}$ ) supplemented with $10 \%$ FBS was added to the bottom of 24-well plates. Cells were seeded at a density of $1 \times 10^{5}$ cells/well into upper inserts and incubated at $37^{\circ} \mathrm{C}$ for $24 \mathrm{~h}$ in $5 \% \mathrm{CO}_{2}$. After $24 \mathrm{~h}$, the non-invading cells were removed from the upper surface of the separating membrane by gentle scrubbing with a cotton swab. Invading cells were fixed in 4\% paraformaldehyde for $5 \mathrm{~min}$ and stained with Giemsa for $9 \mathrm{~min}$. The membranes were mounted on glass slides and manually counted in five random fields using a light microscope. All assays were performed in triplicate.

Knockdown of TF by adenovirus mediated shRNA. We previously constructed adenovirus mediated shRNA against TF (Ad-shRNA-TF) and identified its silencing effect on TF in our previous study (15). To determine the effect of TF expression on A549 cells, we knocked down the TF expression by using Ad-shRNA-TF. Briefly, the A549 cells were plated in 6-well plates and allowed to grow in $2 \%$ FBS media. At $70-80 \%$ confluence, the cells were infected with Ad-shRNA-TF or control at a MOI of $100 \mathrm{vp} / \mathrm{cell}$. Three wells were left uninfected to serve as a negative control. All infections were performed in triplicate. After $12 \mathrm{~h}$, the viral containing media was removed and replaced with fresh media containing $10 \%$ FBS. The silencing effect against TF of the recombinant adenovirus was detected by using RT-PCR and western blot analysis as previously described.

Chemiluminescent secreted alkaline phosphatase assay. The chemiluminescent secreted alkaline phosphatase (SEAP) analysis kit (Clontech, Palo Alto, CA, USA) was used to detect the transcriptional activity of Sp1 in the transfected cells. pTALSEAP vector (negative control), pSEAG2-SEAP vector (positive control) and pSP-SEAP vector, in which SEAP expression is under the control of the Sp1 E-box binding element, were used to transfect the cells, respectively, with Lipofectamine 2000 (Gibco) as described above, except that cells were cultured in 24-well culture plates instead of $60-\mathrm{mm}$ dishes. The culture medium was collected at $24 \mathrm{~h}$ after transfection, was then subjected to the chemiluminescent assay of
SEAP activity using the great EscAPe SEAP kit (Clontech) according to the manufacturer's instructions. The activity data were calculated as: ( $\mathrm{Spl}$ driving vector activity - the negative control activity)/the positive control activity. Each vector was assayed in triplicate and three independent experiments were performed.

Statistical analysis. Results are expressed as the mean \pm SD unless indicated otherwise. ANOVA and Student's t-test were used to determine the statistical significance of differences among experimental groups. Significance was defined as $\mathrm{p}<0.05$.

\section{Results}

Stable transfection of LKB1 in A549 cells. To study the effects of restoring LKB1 expression on A549 lung cancer cells that lack functional LKB1 expression. As described previously, cells were transfected with a mammalian expression vector alone (pcDNA3.1), with pcDNA/Neo carrying human LKB1 cDNA. We selected transfected cells using the Neomicin/G418 resistance gene contained in the pcDNA/Neo expression vector. Sixteen days after selection with G418, there was a clear reduction in the number of colonies expressing wildtype LKB1 compared with those carrying the empty vector. To confirm whether exogenous LKB1 is expressed in A549 transfected cells, we performed RT-PCR and western blot analysis on these transfectants, including clones 1,2 and 3. As shown in lane 5 in Fig. 1, the clone 3 had a higher yield of both LKB1 mRNA and protein. Therefore, the third clone was used to make further experiments in this study.

LKB1 inhibits cell adhesion, migration and invasion in vitro. Compared to the controls, the LKB1 reduced adhesion of A549 cells by $30.44 \%(p<0.05)$. The effects of LKB1 expression on cell migration in vitro are shown in Fig. 3. LKB1-expressing cells exhibited decreased migration. The number of A549/LKB1 cells that migrated through the filter was $54.2 \pm 13.77 / \mathrm{HPF}$, which was significantly lower than that in A549 cells $(97.4 \pm 10.98 / \mathrm{HPF}, \mathrm{p}<0.05)$ (Fig. 2). The effect of LKB1 in regulating the invasive ability of A549 lung cancer cell was examined by using Matrigel invasion assays. The 
A

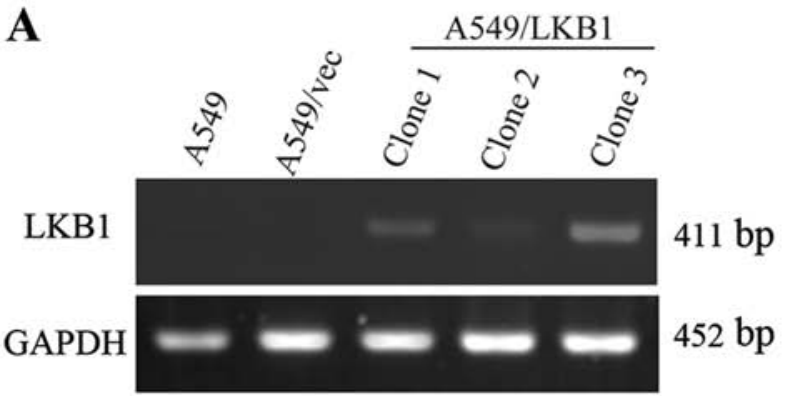

B

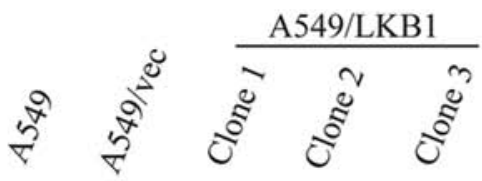

LKB1

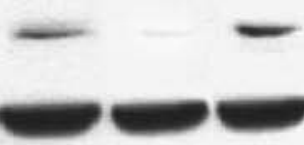

$54 \mathrm{kDa}$

$\beta$-actin
$42 \mathrm{kDa}$

Figure 1. LKB1 expression in the A549/vec and A549/LKB1 cells. Lane 1, control (A549, untransfected cells); lane 2, A549/vector (A549/vec, transfected with vehicle control); lane 3, A549/LKB1 (A549/LKB1, transfected with LKB1) (clone 1); lane 4, A549/LKB1 (clone 2); lane 5, A549/LKB1 (clone 3). (A) LKB1 mRNA expression in the above three A549 clones (RT-PCR). GAPDH was used as control for sample loading. (B) LKB1 protein expression in the above three A549 clones (western blot analysis). $\beta$-actin was used as control for sample loading. These results are representative of at least three independent experiments.

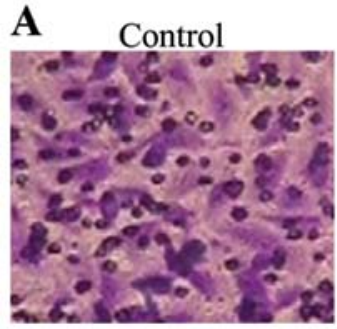

A549/LKB1

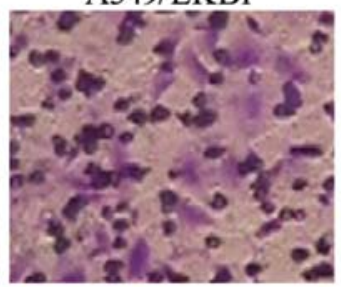

B

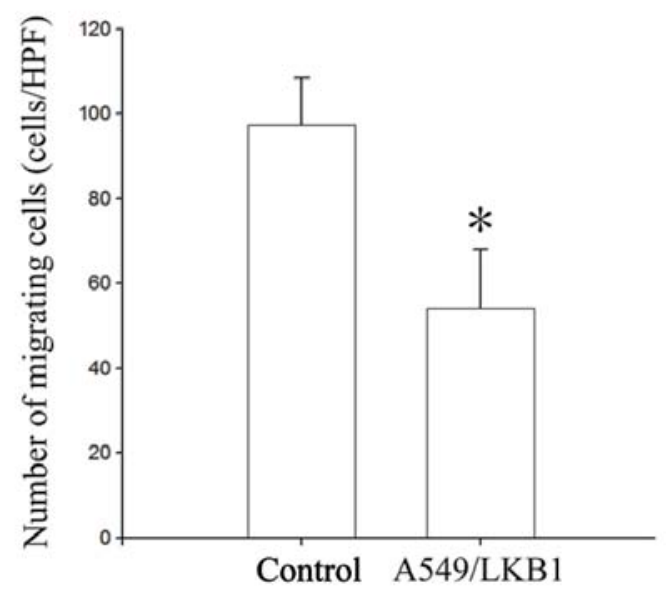

Figure 2. Cell migration of A549 and A549/LKB1 cells in the in vitro migration assays. (A) Light micrograph of migrating cells (x200). (B) The number of cells moving across the filter in the fields per each well. Data are presented as mean $\pm \mathrm{SD}$ of three independent experiments ( $\mathrm{p}<0.05 \mathrm{vs}$. control).

A

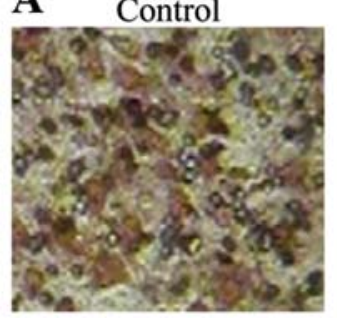

A549/LKB1

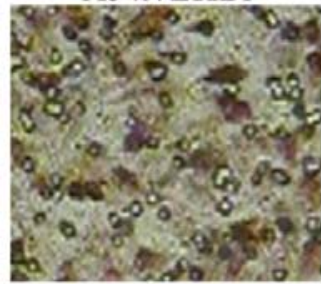

B

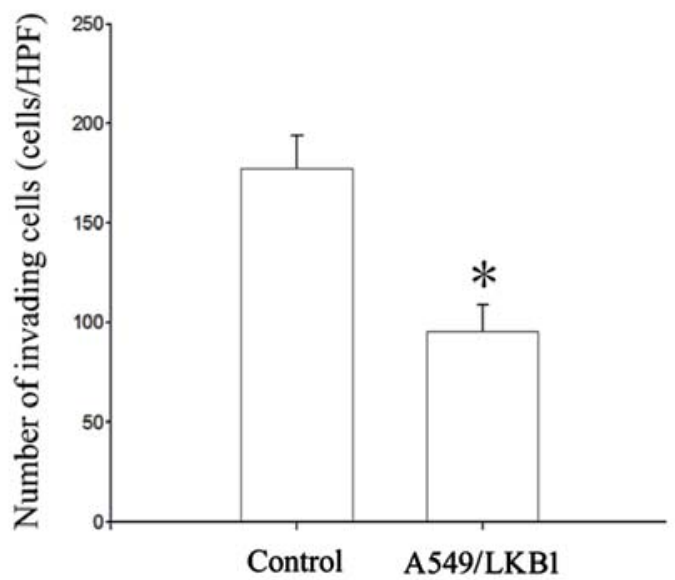

Figure 3. The effects of LKB1 on the invasion of A549 lung cancer cells. Invasiveness was analyzed by Matrigel invasion assay, as described in Materials and methods. (A) Light micrograph of migrating cells (x200). (B) The number of cells invasing through Matrigel and the filter in the fields per each well. Data are presented as mean $\pm \mathrm{SD}$ of three independent experiments ( $\mathrm{p}<0.05 \mathrm{vs}$. control). 
A

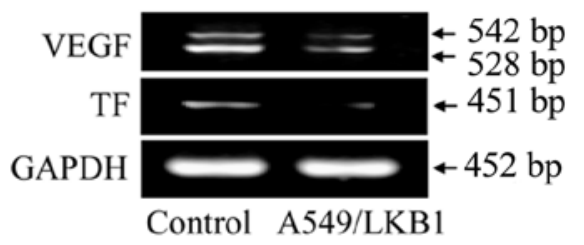

C

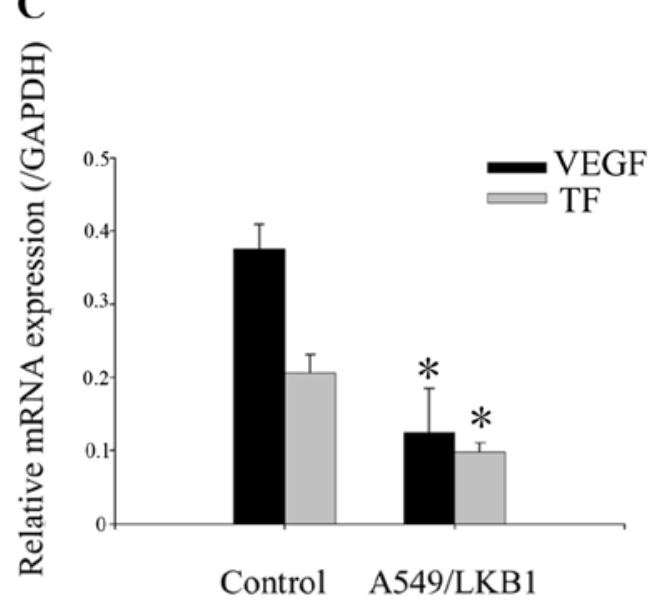

B

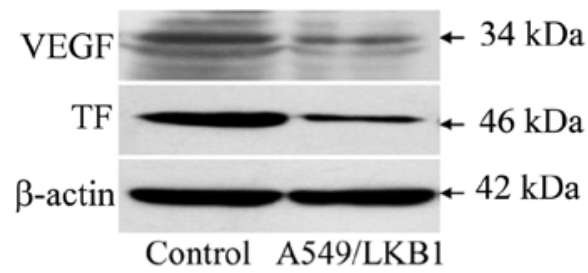

D

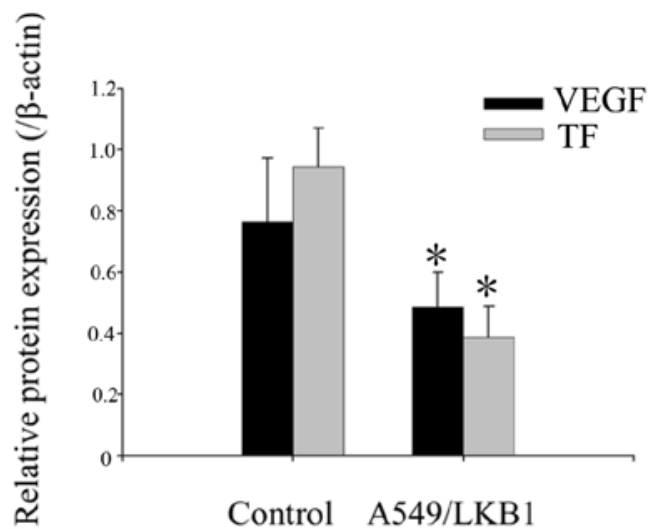

Figure 4. Effect of LKB1 on TF and VEGF expression. (A and B) The mRNA and protein levels of both TF and VEGF were detected by RT-PCR and western blot analysis in A549/vec and A549/LKB1 cells. (C and D) Quantification of mRNA and protein expression levels was expressed as: the ratio of TF, VEGF/GAPDH or $\beta$-actin. Lane 1, control (A549, untransfected cells); lane 2, A549/LKB1(transfected with LKB1). GAPDH or $\beta$-actin are used as control for sample loading $(\mathrm{p}<0.05$ vs. control).
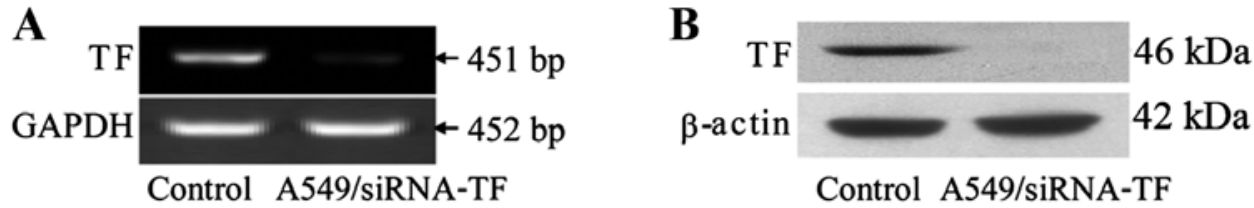

Figure 5. The analysis of TF expression in A549 cells transfected with Ad-shRNA-TF. (A) The mRNA levels of TF was detected by RT-PCR in A549 and A549/siRNA-TF cells. (B) The protein level of TF was detected by western blot analysis in A549 and A549/siRNA-TF cells. Lane 1, control (A549, untransfected cells); lane 2, A549/siRNA-TF (A549 cells transfected with Ad-shRNA-TF at $100 \mathrm{MOI}$ ). GAPDH or $\beta$-actin is used as control for sample loading.

number of A549/LKB1 cells that migrated through the filter was 95.4 $\pm 13.68 / \mathrm{HPF}$. The transfected A549 cells with LKB1 reduced their invasive ability compared to untreated cells $(177.2 \pm 16.43 / \mathrm{HPF}, \mathrm{p}<0.05)$ (Fig. 3). Analysis on the invasive capacity of the analyzed lung cancer cells demonstrated that there was a correlation between LKB1 and invasiveness.

The expression of TF and VEGF can be downregulated by $L K B 1$. To further study the potential correlation between LKB1 and the relevant effectors, we examined the expression of TF and VEGF by using RT-PCR and western blot analysis. Compared with A549 control cells, we showed that LKB1-transfected A549 cells constitutively expressed significantly lower levels of TF and VEGF in both mRNA and protein levels ( $\mathrm{p}<0.05$; Fig. 4$)$.

Adenovirus-mediated shRNA against TF inhibits the expression of TF and results in impaired invasion in A549 cells. Specific gene inhibition in mammalian cells can be achieved by the use of small interfering RNA molecules (siRNA). The silencing effect against TF of the recombinant adenovirus pAd-shRNA-TF was detected by using RT-PCR and western blot analysis. Our results showed that specific reduction in the target protein level was observed after adenoviral infection, and that the reduction in the protein level was correlated with a specific reduction in the mRNA level (Fig. 5). We show that siRNA delivery significantly decreased levels of TF in the lung cancer cell line A549. After transfected with adenoviral vector expressing siRNA against TF, A549 cells showed significantly decreased invasion activity through Matrigel (Fig. 6).

Overexpression of LKB1 inhibits the expression and activity of Sp1. Sp1 is an essential transcription factor for many genes that are key to the regulation of multiple aspects of tumor cell growth and metastasis. To determine whether LKB1 represses Sp1 expression, we examined the effects of LKB1 overexpression on Sp1 expression by using RT-PCR and western blot analysis. We found that overexpressing LKB1 mediated transcriptional 


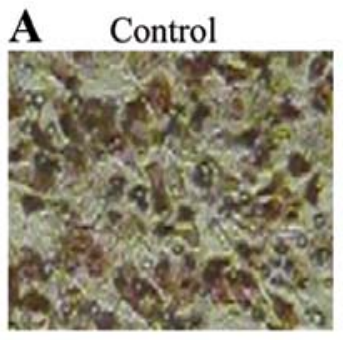

B
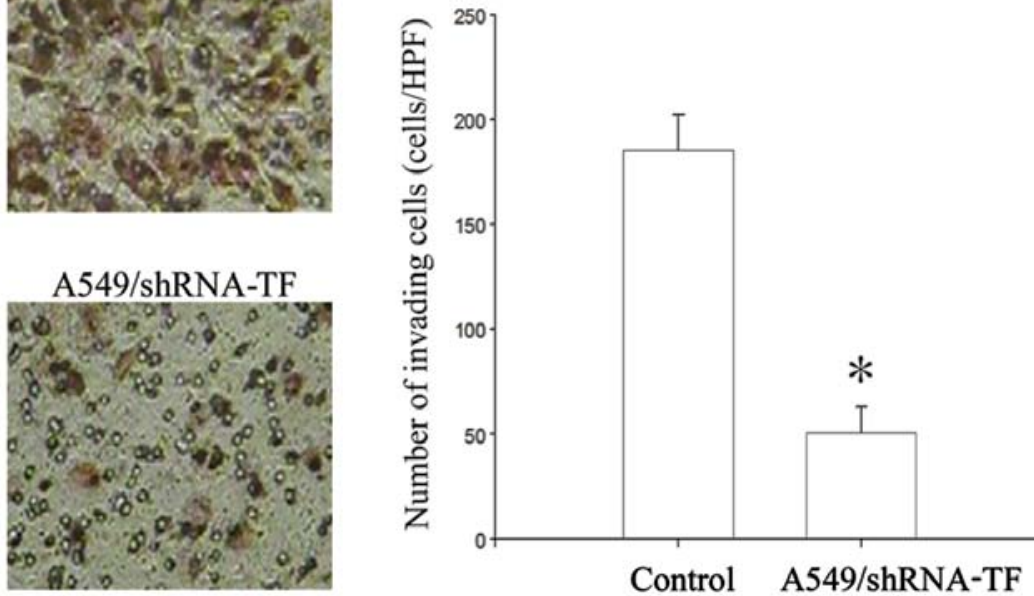

Figure 6. The effects of TF on the invasion of A549 lung cancer cells. Invasiveness was analyzed by Matrigel invasion assay, as described in Materials and methods (A) Light micrograph of migrating cells (x200). (B) The number of cells invading through Matrigel and the filter in the fields per each well. These results are representative of at least three independent experiments. Data are presented as mean \pm SD of three independent experiments ( ${ }^{*} \mathrm{p}<0.05 \mathrm{vs}$. control).

A

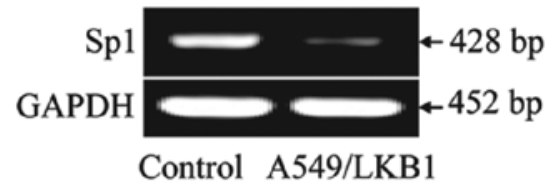

C

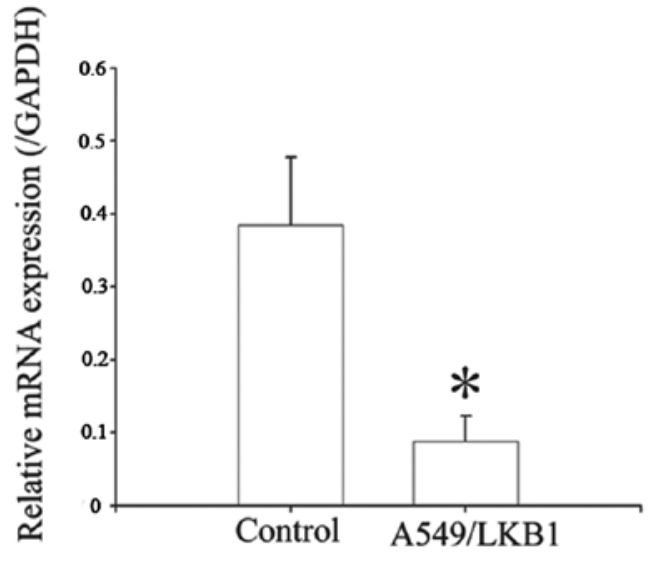

$\mathbf{E}$

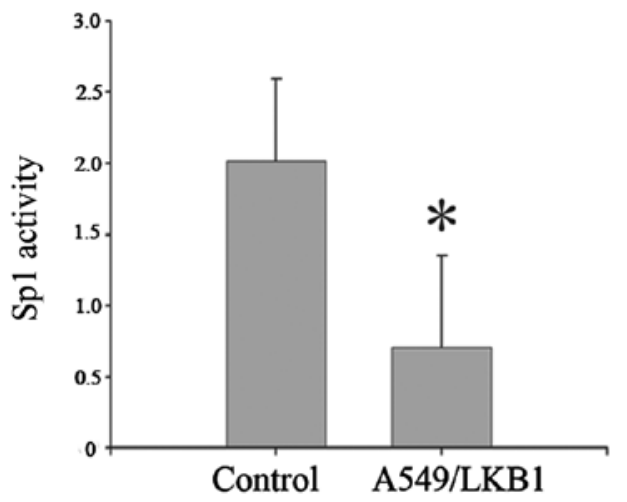

B

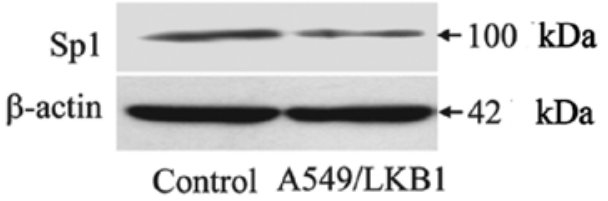

D

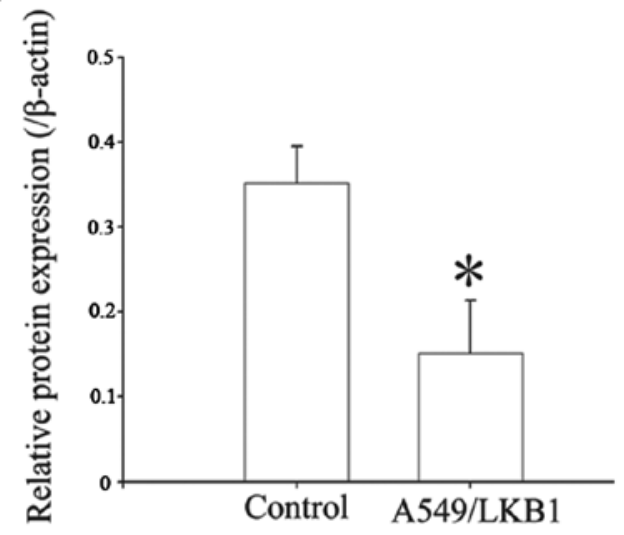

Figure 7. (A and B) Sp1 mRNA or protein expression level in the transfected A549 cells. GAPDH or $\beta$-actin was used as control for sample loading. These results are representative of at least three independent experiments. (C and D) Quantification of mRNA and protein expression levels was expressed as: the ratio of Sp1/ GAPDH or $\beta$-actin. (E) Effect of overexpressing LKB1 on the activity of Sp1 driving SEAP reporter expression in A549 cells. The activity data were calculated as: (Sp1 driving vector expression activity - the negative control activity)/the positive control activity. The data are the means \pm SD from three independent experiments, and each experiment was performed in triplicate ( $\mathrm{p}<0.05 \mathrm{vs}$. control). 
A

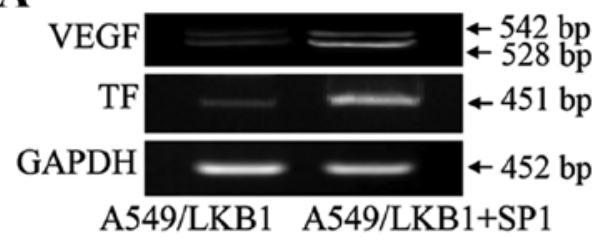

C

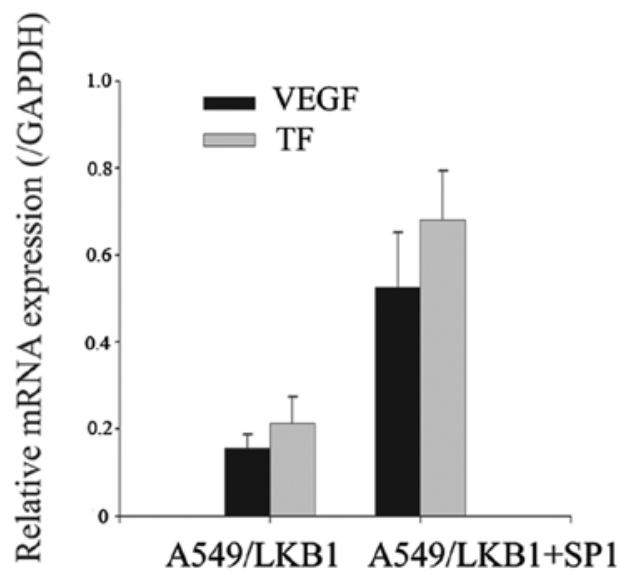

B

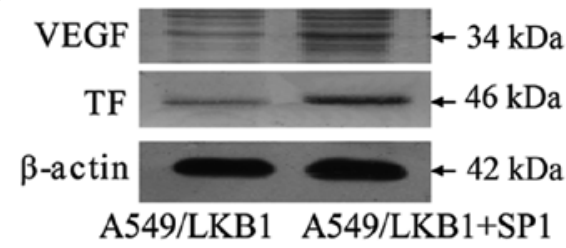

D

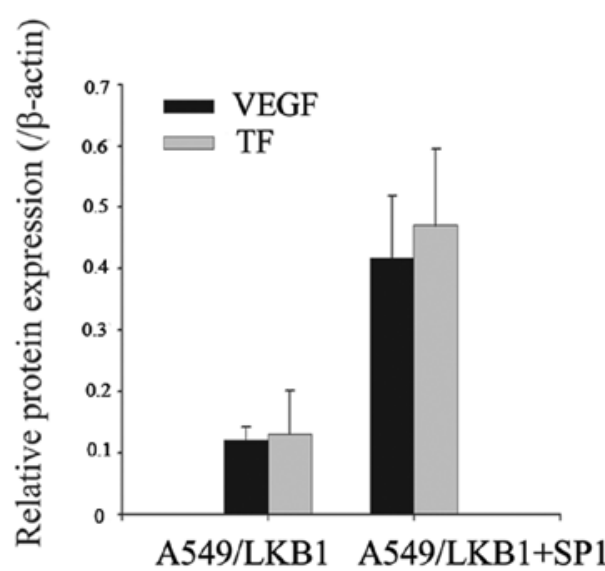

Figure 8. Effect of SP1 on TF and VEGF expression in overexpressed LKB1 cells. (A and B) The mRNA and protein levels of both TF and VEGF were detected by RT-PCR and western blot analysis in A549/LKB1 and A549/LKB1+SP1 cells. GAPDH or $\beta$-actin was used as control for sample loading. (C and D) Quantification of mRNA and protein expression levels was expressed as: the ratio of TF, VEGF/GAPDH or $\beta$-actin. These results are representative of at least three independent experiments $\left({ }^{*} \mathrm{p}<0.05\right.$ vs. control).

repression of $\mathrm{Sp} 1$ gene expression at both the mRNA and protein levels (Fig. 7A and B). To verify this decreased expression of Sp1 protein after overexpressing LKB1, we then investigated the transcriptional activity of Sp1 by means of Sp1 driving the SEAP reporter expression strategy. In this reporter system, expression of SEAP (a secreted form of human placental alkaline phosphatase) in the pSP-SEAP vector is under the control of the E-box enhancer, an Sp1 binding element. Thus, the level of SEAP can well reflect the Sp1 activity in the tested cells. The culture medium was collected at $24 \mathrm{~h}$ after transfecting the cells with the pSP-SEAP vector or the control vectors, and the activities of SEAP in the medium were detected. As shown in Fig. 7E, the transcriptional activity of Sp1 was significantly decreased in the cells overexpressed LKB1.

Spl functions as a positive regulator for TF and VEGF expression. We transfected increasing amounts of Sp1 recombinant plasmid into A549/LKB1 cells and measured the expression of mRNA and protein levels of TF and VEGF gene. Increasing expression of Spl enhanced the TF and VEGF mRNA and protein levels in A549/LKB1 cells (Fig. 8). These results supported the positive role of $\mathrm{Sp} 1$ in the regulation of TF and VEGF.

\section{Discussion}

Tumor development and progression require cell growth, apoptosis resistance, cell invasion and angiogenesis. In our previous study, we observed a suggested tumor-suppressive function for LKB1 in human lung cancer cells proliferation
(9). In the present study, we provide evidence that LKB1 inhibits the migration and invasion of the highly metastatic lung cancer A549 cells. Some studies demonstrated that LKB1 is a critical barrier to pulmonary tumorigenesis, controlling initiation, differentiation and metastasis (16). Consistent with our observation, the anti-invasive property of LKB1 has been demonstrated on human lung carcinoma cells.

In our study, we also showed that TF and VEGF expressions in both mRNA and protein level were significantly downregulated in LKB1-transfected A549 cells in vitro, which may contribute to the inhibition of tumor growth and metastasis. TF plays a major role in promoting tumor metastasis and overexpression of TF has been shown to be associated with the progression and invasion of tumors (17). Its abnormal overexpression has been reported in non-small cell lung cancer and a direct correlation between elevated TF expression and advanced stages of malignancy has been confirmed (18). The present study revealed that knockdown of endogenous TF could suppress the invasiveness of a lung adenocarcinoma cell line in vitro, suggesting that TF plays an important role in tumor invasion. Downregulation of TF expression might lead to the suppression of not only tumor invasiveness but also angiogenesis. Angiogenesis is essential for the growth and metastasis of solid tumors (19). Among the many reported angiogenic factors, VEGF is the most powerful endothelial cell specific mitogens associated with tumor neovascularization. Without vascularization most tumours will not expand and remain non-invasive and do not metastasise $(20,21)$. Various studies indicate that TF plays an essential role in cancer by its ability to upregulate the VEGF gene and thereby improve tumor 
angiogenesis, which is crucial to tumor growth and metastasis $(22,23)$. The pro-angiogenic properties of TF is supported by a significant association between TF expression and a high MVD in various tumors including lung cancer $(23,24)$. The expression of TF contributes to tumor-derived procoagulant activity and the well-known clinical association of malignancy and thrombosis. Such activation may lead to an encapsulation of tumor cells in a platelet-fibrin clot, and arrest in the microcirculation, which is a critically important mechanism in dissemination of tumor cells and may lead to metastasis (25). These findings support the hypothesis that TF expression is positively involved in the mechanisms of human lung cancer development. This study thus provides a mechanistic link between LKB1 tumor suppressor and TF gene expression.

Invasive potential of LKB1-null cells is, at least in part, mediated by SP1 because most cells with downregulation of SP1 levels showed decreased cellular migration and invasion. Because Sp1 is an essential transcription factor for many genes that are key to the regulation of multiple aspects of tumor cell survival, growth, and angiogenesis, abnormal Sp1 expression and activation may contribute to lung cancer development and progression $(26,27)$. Enforced LKB1 expression repressed Sp1 expression at the promoter activity, mRNA, and protein levels. Sp1 promoter contains putative Sp1 binding sites, Sp1 overexpression may result from autotransactivation of its own promoter. A region within the proximal Sp1 promoter was identified to have overlapping LKB1 and Sp1-binding sites, to which LKB1 and Sp1 compete for binding (28). Sp1 positively regulated its own promoter, whereas LKB1 did the opposite. Our data suggests that disruption of LKB1-mediated negative regulation contributes to the molecular events of Sp1 overexpression and to the development and progression of human lung cancer. Furthermore, constitutive activation of the transcription factor Spl plays a critical role in VEGF and TF expression $(29,30)$. We also proved that $\mathrm{Spl}$ plays an important role in the regulation of VEGF and TF expression which is essential for the invasion and metastasis of lung cancer. We transfected exogenous Sp1 into A549/LKB1 cells and found that increasing expression of Spl enhanced the TF and VEGF mRNA and protein levels in A549/LKB1 cells. Therefore, the LKB1-reduced invasiveness of human lung cancer cells was found to correlate with downregulation of TF and VEGF expression and was thought to be regulated by blocking the SP1 activation and/or its expression.

In conclusion, we identified that overexpression of LKB1 inhibited lung cancer invasion in human lung cancer A549 cells, and the antitumor activity of LKB1 gene is correlated with suppression of TF and VEGF, which partly depend on the activation of the transcription factor Spl. To our knowledge the present study is the first to demonstrate the role of LKB1 in regulating TF-mediated invasion on A549 cells. TF may be a viable therapeutic target for human cancers with LKB1 mutations. These results suggest that $\mathrm{LKB} 1$ represents a potential anti-metastatic gene therapy and this new beneficial effect may expand future research on anticancer properties of LKB1 in vitro and in vivo. Further validation of these results will require studies in more cell lines, in vivo studies, and additional observations on LKB1 downstream effects. Collectively, our data provide a novel molecular mechanism for the antitumor activity of LKB1 and may help further improve its effectiveness in controlling lung cancer growth and metastasis.

\section{Acknowledgements}

This study was supported by National Natural Science Foundation of China (no. 81101777). We thank Dr Qingzhi Xu from the Academy of Military Medical Sciences of China (Beijing, RPB, China) for providing us with pcDNA 3.1 expression vector. We thank Dr J. Marvel and Dr Y. Leverrier from Inserm of France (Lyon, France) for providing us with pBSK-SP1-AN plasmid.

\section{References}

1. Bennett $\mathrm{A}$ and White $\mathrm{J}$ : Improving care and quality of life for patients with lung cancer. Nursing Stand 28: 50-58, 2013.

2. Gutierrez-Fernandez A, Fueyo A, Folgueras AR, et al: Matrix metalloproteinase- 8 functions as a metastasis suppressor through modulation of tumor cell adhesion and invasion. Cancer Res 68: 2755-2763, 2008.

3. Sugahara KN, Hirata T, Tanaka T, et al: Chondroitin sulfate E fragments enhance CD44 cleavage and CD44-dependent motility in tumor cells. Cancer Res 68: 7191-7199, 2008.

4. Ghaffar H, Sahin F, Sanchez-Cepedes M, et al: LKB1 protein expression in the evolution of glandular neoplasia of the lung. Clin Cancer Res 9: 2998-3003, 2003.

5. Jimenez AI, Fernandez P, Dominguez O, Dopazo A and Sanchez-Cespedes M: Growth and molecular profile of lung cancer cells expressing ectopic LKB1: down-regulation of the phosphatidylinositol 3'-phosphate kinase/PTEN pathway. Cancer Res 63: 1382-1388, 2003.

6. Marcus AI and Zhou W: LKB1 regulated pathways in lung cancer invasion and metastasis. J Thorac oncol 5: 1883-1886, 2010.

7. Liang $X$, Nan KJ and Xu QZ: Effect of small interfering RNA-induced LKB1 gene silencing on the biological behavior of lung carcinoma cells. Nan Fang Yi Ke Da Xue Xue Bao 27: 1303-1306, 2007 (In Chinese).

8. Liang X, Nan KJ, Li CL, Yao Y, Tian T and Wang SH: Effect of transfection of LKB1 on biological behavior of human lung adenocarcinoma cells. Xi Bao Yu Fen Zi Mian Yi Xue Za Zhi 24: 1140-1142, 2008 (In Chinese).

9. Liang X, Nan KJ, Li ZL and Xu QZ: Overexpression of the LKB1 gene inhibits lung carcinoma cell proliferation partly through degradation of c-myc protein. Oncol Rep 21: 925-931, 2009.

10. Ylikorkala A, Rossi DJ, Korsisaari N, et al: Vascular abnormalities and deregulation of VEGF in Lkb1-deficient mice. Science 293: 1323-1326, 2001.

11. Brugarolas $\mathrm{J}$ and Kaelin WG Jr: Dysregulation of HIF and VEGF is a unifying feature of the familial hamartoma syndromes. Cancer Cell 6: 7-10, 2004.

12. Nakasaki T, Wada H, Shigemori C, et al: Expression of tissue factor and vascular endothelial growth factor is associated with angiogenesis in colorectal cancer. Am J Hematol 69: 247-254, 2002.

13. Zhang J, Ding J, Zhang X, Shao X and Hao Z: Regulation of vascular endothelial growth factor (VEGF) production and angiogenesis by tissue factor (TF) in SGC-7901 gastric cancer cells. Cancer Biol Ther 4: 769-772, 2005.

14. Koomagi R and Volm M: Tissue-factor expression in human non-small-cell lung carcinoma measured by immunohistochemistry: correlation between tissue factor and angiogenesis. Int J Cancer 79: 19-22, 1998.

15. Li ZL, Xu WJ, Tian PX, et al: Recombinant adenovirus-mediated RNA silencing of tissue factor expression in human islet: an in vitro study. Nan Fang Yi Ke Da Xue Xue Bao 27: 1299-1302, 2007 (In Chinese).

16. Ji H, Ramsey MR, Hayes DN, et al: LKB1 modulates lung cancer differentiation and metastasis. Nature 448: 807-810, 2007.

17. Xu C, Gui Q, Chen W, et al: Small interference RNA targeting tissue factor inhibits human lung adenocarcinoma growth in vitro and in vivo. J Exp Clin Cancer Res 30: 63, 2011.

18. Minamiya Y, Matsuzaki I, Sageshima M, et al: Expression of tissue factor mRNA and invasion of blood vessels by tumor cells in non-small cell lung cancer. Surg Today 34: 1-5, 2004.

19. Mohammadi B, Haghpanah V and Larijani B: A stochastic model of tumor angiogenesis. Comput Biol Med 38: 1007-1011, 2008.

20. Vokes E, Herbst R and Sandler A: Angiogenesis inhibition in the treatment of lung cancer. Clin Adv Hematol Oncol 4: 1-10, 2006. 
21. Wang S, Liu H, Ren L, Pan Y and Zhang Y: Inhibiting colorectal carcinoma growth and metastasis by blocking the expression of VEGF using RNA interference. Neoplasia 10: 399-407, 2008.

22. Rickles FR, Shoji M and Abe K: The role of the hemostatic system in tumor growth, metastasis, and angiogenesis: tissue factor is a bifunctional molecule capable of inducing both fibrin deposition and angiogenesis in cancer. Int J Hematol 73: 145-150, 2001

23. Regina S, Rollin J, Blechet C, Iochmann S, Reverdiau P and Gruel Y: Tissue factor expression in non-small cell lung cancer: relationship with vascular endothelial growth factor expression, microvascular density, and K-ras mutation. J Thorac Oncol 3: 689-697, 2008.

24. Poon RT, Lau CP, Ho JW, Yu WC, Fan ST and Wong J: Tissue factor expression correlates with tumor angiogenesis and invasiveness in human hepatocellular carcinoma. Clin Cancer Res 9 5339-5345, 2003

25. Regina S, Valentin JB, Lachot S, Lemarie E, Rollin J and Gruel Y: Increased tissue factor expression is associated with reduced survival in non-small cell lung cancer and with mutations of TP53 and PTEN. Clin Chem 55: 1834-1842, 2009.
26. Han S and Roman J: COX-2 inhibitors suppress integrin alpha5 expression in human lung carcinoma cells through activation of Erk: involvement of Sp1 and AP-1 sites. Int J Cancer 116: 536-546, 2005

27. Zhang J, Jia Z, Li Q, et al: Elevated expression of vascular endothelial growth factor correlates with increased angiogenesis and decreased progression-free survival among patients with low-grade neuroendocrine tumors. Cancer 109: 1478-1486, 2007.

28. Lutzner N, De-Castro AJ and Rosl F: Gene expression of the tumour suppressor LKB1 is mediated by Sp1, NF-Y and FOXO transcription factors. PLoS One 7: e32590, 2012.

29. Shi Q, Le X, Abbruzzese JL, et al: Constitutive Sp1 activity is essential for differential constitutive expression of vascular endothelial growth factor in human pancreatic adenocarcinoma. Cancer Res 61: 4143-4154, 2001.

30. Ishibashi H, Nakagawa K, Onimaru M, et al: Sp1 decoy transfected to carcinoma cells suppresses the expression of vascular endothelial growth factor, transforming growth factor beta1, and tissue factor and also cell growth and invasion activities. Cancer Res 60: 6531-6536, 2000. 